\title{
Enhance the efficiency of the internal threads cutting process with taps by applying ultrasonic vibrations
}

\author{
Valery Golovkin ${ }^{1, *}$, Oksana Batishcheva ${ }^{1}$, and Valery Papshev ${ }^{1}$ \\ ${ }^{1}$ Samara State Technical University, 443100, ul. Molodogvardeyskay, 244, Samara, Russian \\ Federation
}

\begin{abstract}
The paper considers a technological process of an advanced method of cutting internal threads using ultrasonic vibrations. A special ultrasonic device is shown, which makes it possible to realize this method of ultrasonic processing. The paper also presents research findings of ultrasonic vibrations influence on the process of cutting internal threads.
\end{abstract}

\section{Introduction}

Currently, one of the promising areas for increasing the efficiency of various machining processes is the development and implementation of qualitatively new technologies. These technologies are based on the combined effect of several types of energy. Along with other methods of physico-chemical processing, various processes of ultrasonic technology are becoming increasingly widespread: for example, blade processing with the introduction of forced ultrasonic vibrations into the cutting zone [1]. It should be noted that when processing with a multi-blade tool, the effect of ultrasonic vibrations is more pronounced. The use of ultrasound turned out to be particularly effective when cutting internal threads with taps in workpieces made from hard-to-work materials. At the same time, it was established that the imposition of forced ultrasonic vibrations on the tap allows:

- $\quad$ to increase the labor productivity and efficiency of the tool;

- $\quad$ to improve the qualitative characteristics of the surface layer;

- to increase the accuracy of the threads to be cut.

\section{Equipment and methodology of the experiment}

In order to determine the effectiveness of the influence of ultrasonic vibrations on the thread cutting process, comparative studies were carried out using special ultrasonic threading devices developed at Samara State Technical University [1].

The ultrasonic device used in this research is equipped with a piezoceramic transducer and has a small size and mass. It does not require the use of water cooling, which greatly enhances the usability and versatility of this device. This ultrasonic device is provided with

\footnotetext{
${ }^{*}$ Corresponding author: valeriigolovkin1962@mail.ru
} 
compensation for possible axis misalignment of the tap and the hole being processed. There is also a setting for the required depth of tapping.

Fig. 1 presents a general view of a version of the ultrasonic device. Using this ultrasonic device, comparative studies of the influence of ultrasound on the process of cutting internal threads were undertaken. Whereas, the ultrasonic vibrations were transmitted to the tap axially.

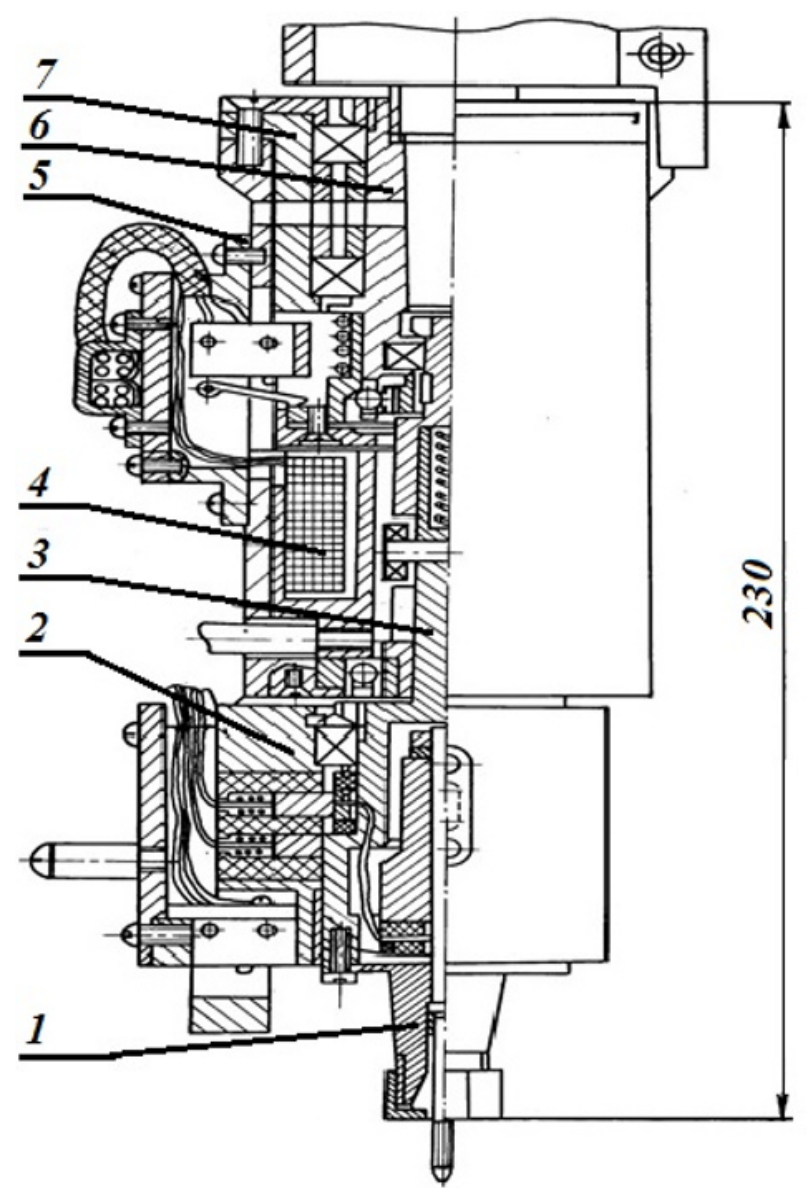

Fig. 1. Ultrasonic device for cutting threads M3-M8.

Thread cutting was implemented in the speed range from 0.6 to 3 meters per minute, the amplitude of ultrasonic vibrations ranged from 0 to $6 \mu \mathrm{m}$. The processing was carried out at a resonance frequency within $19-21 \mathrm{kHz}$.

This device contains a piezoceramic transducer 1 and a current-carrying unit 2 set in a telescopic system 3. This system enables to compensate for the setting error of the processed workpiece on the machine-tool. The assembly 5 performs a short-time spindle reverse when the safety clutch is actuated. In this device, all assemblies are located in a stationary housing 7. The drive shaft 6 has a tapered mounting bore to be fitted to the machine-tool spindle. The device also has an electromagnetic clutch 4 . It is designed for smooth and fine adjustment of the safety device at the maximum permissible torque. 


\section{Results}

The results obtained in the course of the experimental researches are given below. Fig. 2 illustrates an oscillogram characterizing the cutting torque - CT when threading M8 in a titanium alloy VT9.

The analysis of the obtained results showed that when the ultrasonic vibrations are applied to the tap axially with amplitude of $5 \mu \mathrm{m}$, the cutting torque decreases by $30-40 \%$.

It should be noted that the decrease in the values of the cutting torque is associated, first of all, with a decrease in the coefficient of friction on the cutting and calibrating teeth of the tap. It is also associated with the change in the vectors of forces on the cutting edges, the activation of the technological environment and other factors. As a result, the conditions of the cutting process are improved, which in turn leads to a significant improvement of the tool life period.

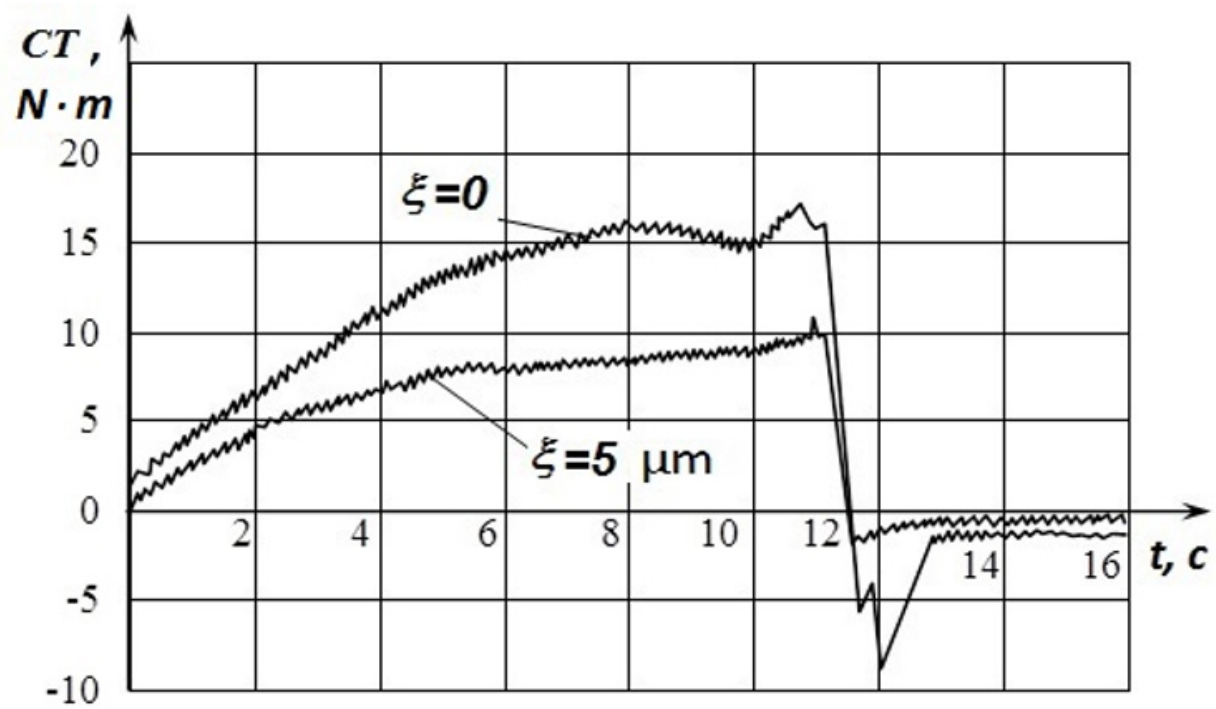

Fig. 2. Oscillogram CT when cutting thread M8 in a titanium alloy VT9.

It worth mentioning the fact that when threading in a blind hole at the beginning of unscrewing the tap, a torque leap occurs because of cutting the roots of chips or falling of cut chips under the backed-off cutting teeth of the tap. This sometimes leads to the chipping of the cutting part. As it is seen on the above given oscillogram when processing with ultrasonic vibrations, the values of the torque during unscrewing at the initial moment are much less than when cutting threads without ultrasound.

Taking into consideration this fact, special researches have been conducted on the effect of ultrasound on the torque of unscrewing when threading in blind holes. Fig. 3 outlines the results of a study on the impact of the amplitude of oscillations $\xi$ on $C T_{\text {unscrew. }}$. when processing alloy VT9 with a set of taps M8×1,25 and taps with TiN coating.

It can be seen in the graphs, an increase in the amplitude of ultrasonic vibrations of up to $5 \mu \mathrm{m}$ leads to a reduction in the torque of unscrewing. The coating of taps with titanium nitride does not have a significant effect on the torque values of unscrewing. It should be pointed out that the use of ultrasound leads to a significant increase in the efficiency of taps due to reduced forces, the reduction of wear and chipping of the cutting teeth of the tap. 


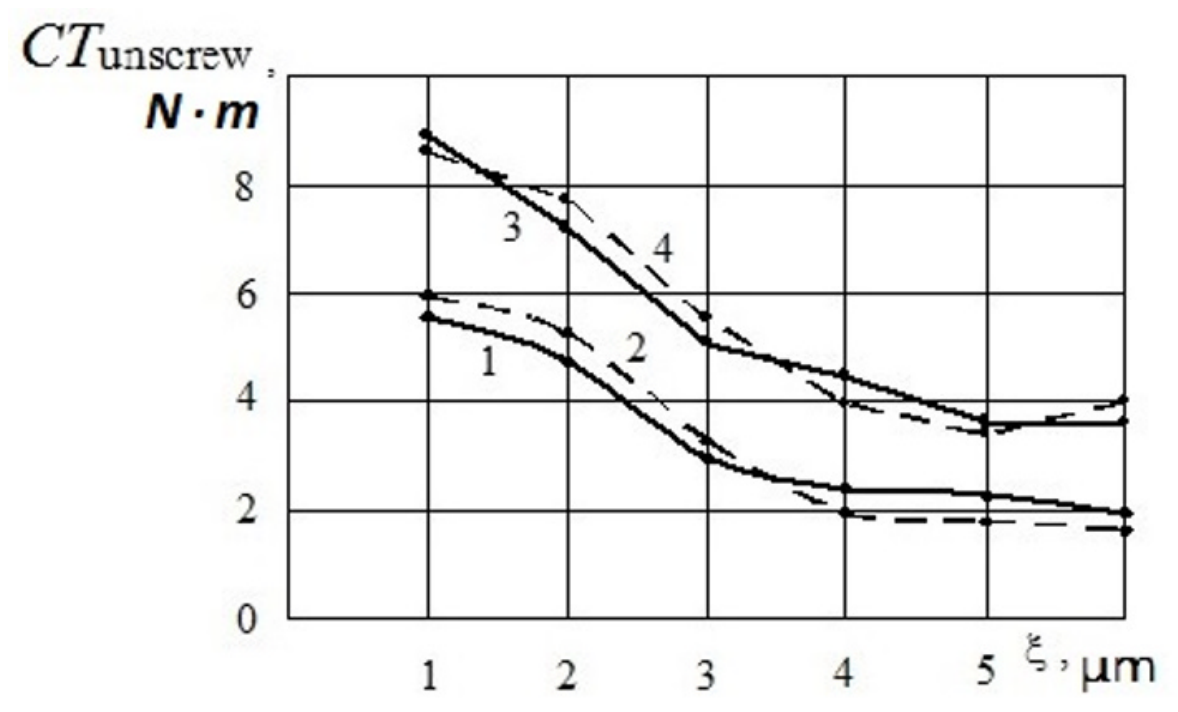

Fig. 3. Influence of the amplitude of oscillations on $C T_{\text {unscrew. }}$ when processing alloy VT9 with the tap M8 $\times 1,25: 1$ - the first tap from the kit; 2 - the first tap from the set with TiN coating; 3 - the third tap from the kit; 4 - the third tap from the kit with TiN coating.

\section{Conclusions}

The research has shown that using the forced ultrasonic vibrations, when cutting the internal threads with taps, allows reducing the rotational torque of cutting to $30-40 \%$. This makes it possible to increase the processing efficiency and working capacity of the tool.

\section{References}

1. B.L. Shtrikov, V.V. Golovkin, V.G. Shouvayev, I.V. Shuvayev, Improving the efficiency of threaded joints by using ultrasound when processing and assembling (Moscow: Engineering, 2009) 